\title{
Tolerability of atypical neuroleptics and acetylcholinesterase inhibitors when treating neuropsychiatric symptoms in Parkinson's disease
}

This article was published in the following Dove Press journal:

Journal of Parkinsonism \& Restless Legs Syndrome

25 September 2012

Number of times this article has been viewed

\author{
Christopher Tolleson' \\ John Fang' \\ Pengcheng $\mathrm{Lu}^{2}$ \\ Huiyun $\mathrm{Wu}^{2}$ \\ Thomas Davis' \\ 'Movement Disorders, Department \\ of Neurology, ${ }^{2}$ Department of \\ Biostatistics, Vanderbilt University, \\ Nashville, TN, USA
}

\begin{abstract}
Background: Treating neuropsychiatric problems in Parkinson's disease (PD) remains challenging. Only rivastigmine has US Food and Drug Administration approval for the treatment of PD dementia. Some studies have shown that atypical neuroleptics adequately improve hallucinations while others suggest that side effects and increased mortality negate benefit. We utilized a chart review to assess the tolerability and efficacy of commonly used agents for neuropsychiatric symptoms in PD.

Methods: We reviewed patients with a PD diagnosis in our clinic database who were being treated with antidementia agents (donepezil, galantamine, rivastigmine, memantine) or atypical neuroleptics (quetiapine, olanzapine, clozapine) by two PD specialists over 13 years. We determined tolerability by therapy retention in comparison with the accepted gold standard, ie, rivastigmine for dementia and clozapine for hallucinations. Clinician documentation of patient/ caregiver or personally perceived clinical improvement was recorded to define therapeutic response. Secondary outcome measures were death, treatment-limiting side effects, and weight change.
\end{abstract}

Results: A total of 171 PD patients met criteria for study inclusion. All medications were similarly retained in comparison with the standard, with the exception of donepezil which was significantly better $(P<0.02)$ than rivastigmine. Using the documentation metric, no medication was statistically superior to its standard. Olanzapine approached significance because it was inferior to the clozapine standard $(P<0.08)$. Secondary outcome measures were similar among medications.

Conclusion: We conclude that, as a group, acetylcholinesterase inhibitors, atypical neuroleptics, and memantine are well tolerated in treating either cognitive difficulties or hallucinations in PD. They appear to offer variable levels of clinical benefit, as well per documented therapeutic responses. Side effects, death, and weight change were not significantly different among medications.

Keywords: Parkinson's disease, atypical neuroleptics, acetylcholinesterase inhibitors, dementia, hallucinations

\section{Introduction}

Parkinson's disease (PD) is the second most common neurodegenerative disorder, affecting millions of people worldwide, with most older than 60 years. ${ }^{1}$ It is diagnosed clinically by features of asymmetric resting tremor, rigidity, bradykinesia, and postural instability. The main PD treatments principally alleviate these motor symptoms by augmenting dopaminergic function in the basal ganglia. However, nonmotor symptoms are often much more debilitating, and treatments are less well established. ${ }^{2-5} \mathrm{PD}$ patients suffer from a variety of neuropsychiatric complaints, including psychosis at
Correspondence: Christopher M Tolleson Movement Disorders, Department of Neurology, A-0I I 8 Medical Center North Mail Room, II6I 21 st Avenue South, Nashville, TN 37232-255I I, USA

$\mathrm{Tel}+\mathrm{I} 6159362025$

Fax +16159361229

Email christopher.tolleson@vanderbilt. edu 
rates of $30 \%-40 \%$ and dementia at approximately $30 \% .^{4-6}$ Several agents have been used in attempts to alleviate both these symptoms. Unfortunately, there is a paucity of available comparative efficacy data. ${ }^{7}$

Typically, acetylcholinesterase inhibitors and memantine are the agents used clinically for PD dementia. Donepezil and rivastigmine have both been shown in several studies to have a mild to modest benefit in cognition. ${ }^{8-12}$ However, only rivastigmine (Exelon ${ }^{\circledR}$; Novartis Pharmaceuticals; Basel, Switzerland) has received approval for this indication from the US Food and Drug Administration in contrast with the other agents which have been approved in Alzheimer's disease. Although not approved by the Food and Drug Administration for PD, atypical neuroleptics are often favored in the treatment of psychosis when hallucinations are most prominent. ${ }^{5}$ Atypical neuroleptics as a group have been shown to cause fewer extrapyramidal symptoms than the typical antipsychotics, which mainly target the D2 receptor. ${ }^{13}$ The atypical neuroleptic, clozapine, has generally been regarded as the most effective, based upon two double-blind, placebo-controlled studies, and another atypical neuroleptic, quetiapine, may also be beneficial in some patients. ${ }^{7,14-16}$ Published results for quetiapine have been less consistent. ${ }^{17}$ A third atypical neuroleptic, olanzapine, has lower efficacy, and its utility has been limited by worsening PD motor function. ${ }^{18-20}$

Many studies, including some related to CATIE (Clinical Antipsychotic Trials in Intervention Effectiveness), raise concerns that any atypical neuroleptic benefits are offset by side effects and intolerability. ${ }^{21}$ In these studies, olanzapine and quetiapine were associated with a higher degree of cardiovascular morbidity ${ }^{22}$ and weight gain, ${ }^{23}$ while the class as a whole was shown to increase the odds of death by 1.3-1.9 times that in the nontreatment group. ${ }^{24}$

In this study, our goal was to compare the use of dementia and psychosis medications in our clinical practice. We examined the use of acetylcholinesterase inhibitors, memantine, and atypical neuroleptics to delineate better the tolerability and efficacy of each drug. We also sought to examine better the level of side effects for each drug to determine the extent to which side effects offset symptomatic benefit. Because acetylcholinesterase inhibitors and atypical neuroleptics are often used in elderly PD patients suffering from multiple comorbidities and moderately severe cognitive dysfunction, it was felt impractical to perform formalized neuropsychologic testing in this population due to "floor" effects; patients generally cannot cooperate sufficiently to provide meaningful data. We employed a model whereby clozapine was designated as the reference atypical neuroleptic, and rivastigmine was designated as the reference acetylcholinesterase inhibitor. Each medication was compared statistically with its reference standard for both symptom improvement and tolerability. This design created a practical method for identifying potential differences between commonly used treatments for dementia and psychosis in PD, while maintaining the focus on severely affected patients.

\section{Materials and methods}

We conducted a retrospective analysis of patients taking donepezil, galantamine, rivastigmine, memantine, quetiapine, olanzapine, or clozapine treated by two movement disorder specialists (JF and TD) at Vanderbilt University Hospital. The study protocol was approved by the Vanderbilt institutional review board. We examined medical records data stored in a clinical database named StarPanel which held all patient information from Vanderbilt University from January 1, 1998 until the present. Patients who carried the diagnosis of PD were included in the study up to the date of October 31, 2010. Patients were selected from those patients seen by JF or TD by inputting the search terms "rivastigmine", "Exelon ${ }^{\circledR} "$, "donepezil", "Aricept ${ }^{\circledR} "$, "galantamine", "Razadyne ${ }^{\circledR} "$, "memantine", "Namenda ${ }^{\circledR} "$, "quetiapine", "Seroquel ${ }^{\circledR} "$, olanzapine, "Zyprexa ${ }^{\circledR} "$, "Clozaril ${ }^{\circledR} "$, and "clozapine" into the search engine in the database. This large group of patients underwent chart review to determine if the treating physician indeed felt they had idiopathic PD, and whether the selected agents were used for dementia or hallucinations. Patients were diagnosed by our movement specialists with PD if they had two of the four cardinal motor features (resting tremor, bradykinesia, rigidity, postural instability) as well as a robust response to L-dopa. Patients without L-dopa responsiveness or these cardinal features were excluded from further analysis, with the exception of eight patients with documented partial L-dopa responsiveness and the aforementioned clinical features.

Tolerability was defined as drug retention time as compared with the gold standard for dementia (rivastigmine) and hallucinations (clozapine). Efficacy was defined by documented clinical benefit from either the patient/caregiver or the physician. These data were not available in the chart for every patient. These patients were not included in the later analysis for this metric. Secondary outcome measures included weight change, death, and documented treatmentlimiting side effects. Death was only recorded when there was a documented date of death, despite several instances where elderly frail patients were lost to follow-up for years 
at a time. Weight change was measured using the available visit data corresponding to initiation of medication until the last documented weight at the visit where the medication was discontinued or the last documented clinic visit. Side effects were determined by documented patient/caregiver complaint and assigned to several categories, ie, worsening motor function, worsening cognition, somnolence/sedation, gastrointestinal side effects, neutropenia, and other.

We tabulated the number of months patients were on particular drugs to determine retention time. Those who entered the study cohort less than 12 months before the end date and did not stop a particular drug prematurely due to side effects or lack of benefit were censored. The cohort with censored data was then analyzed for retention time using Kaplan-Meier estimators. Log rank test statistics and $P$ values were computed to compare the difference in retention time between a drug group and a reference group. The $P$ values were adjusted for multiple comparisons using the Bonferroni method. The gold standard drugs were used as reference for comparisons for their respective indications: rivastigmine for cognition drugs (donepezil, galantamine, memantine) and clozapine for hallucination drugs (olanzapine, quetiapine). Efficacy or treatment benefit was evaluated as the proportion of those (patients/caregivers or physicians) reporting documented positive benefit from the drug. The difference between a drug and the reference drug was tested using Chi-square analysis. The benefit reported by physicians or patients/caregivers was analyzed separately. Corrections for multiple comparisons again utilized the Bonferroni method. Because a drug was evaluated for treatment benefit by both physicians and patients, a Kappa test was performed to examine whether the two evaluations agreed with each other for each individual drug. All tests were two-tailed, with $P<0.05$ deemed as significant. All computations were done using $\mathrm{R}$ (http://cran.r-project.org/).

\section{Results}

A total of $171 \mathrm{PD}$ patients met the criteria for inclusion. This group reflected a fairly typical subset of the national PD population, with a higher percentage of men (64.91\%) and a median age of onset of 60 years. The median time from onset until treatment with a neuropsychiatric medication was 111 months (Table 1). The number of patients treated with each of the targeted medications was as follows: clozapine $(n=30)$, olanzapine $(n=12)$, quetiapine $(n=114)$, memantine $(n=53)$, galantamine $(n=2)$, donepezil $(n=63)$, and rivastigmine $(n=73)$. Patients received a variety of different medication combinations such that $44.44 \%$ were on a
Table I Patient characteristics

\begin{tabular}{|c|c|}
\hline & $\begin{array}{l}\text { Descriptive } \\
\text { numbers }\end{array}$ \\
\hline \multicolumn{2}{|l|}{ Gender } \\
\hline Male & $64.91 \%$ \\
\hline \multicolumn{2}{|l|}{ Age at diagnosis* } \\
\hline Mean \pm standard deviation & $59.14 \pm 10.74$ \\
\hline Median with interquartile range & $60(58-67)$ \\
\hline \multicolumn{2}{|c|}{ Time from diagnosis to onset of medication use (months)* } \\
\hline Mean \pm standard deviation & $130.12 \pm 84.64$ \\
\hline Median with interquartile range & $111(72-180)$ \\
\hline \multicolumn{2}{|l|}{ Comorbidities } \\
\hline Neurologic comorbidities ${ }^{\dagger}$ & $12.28 \%$ \\
\hline Cardiac comorbidities ${ }^{\ddagger}$ & $32.75 \%$ \\
\hline Metabolic/nutritional comorbidities ${ }^{*}$ & $7.02 \%$ \\
\hline \multicolumn{2}{|l|}{ Diabetes mellitus } \\
\hline Before Parkinson's disease & $4.68 \%$ \\
\hline During Parkinson's disease & $2.92 \%$ \\
\hline Prescribed both dementia and antipsychotic agents* & $44.44 \%$ \\
\hline
\end{tabular}

Notes: *Patients were labeled as unknown and not included in analysis if exact values were not known; ${ }^{\dagger}$ comorbidities included traumatic brain injury, intracranial bleed, epidural abscess, stroke/transient ischemic attack, mental retardation, hydrocephalus/shunting, seizure, syncope, Guillain Barre syndrome; *coronary artery disease, hypertension, atrial fibrillation, myocardial infarction, valve abnormality, arrhythmia, open heart surgery, ischemic cardiac myopathy; *hyperthyroidism, hypothyroidism, hyponatremia, BI2 deficiency.

combination of dementia and antipsychotic medication for at least some time during the studied period. Often different medications from the same class were used at different points in the patient's treatment. With only a few exceptions, dementia symptoms were treated with acetylcholinesterase inhibitors and memantine, whereas hallucinations were treated with atypical neuroleptics.

We used retention rate as compared with the accepted gold standard to assess tolerability (Table 2). When comparing cognitive agents with rivastigmine, approved by the Food and Drug Administration, donepezil was found to be better retained and potentially better tolerated (donepezil median retention time $=37.5$ versus rivastigmine median retention time $=14$, $\log$ rank $6.2, P<0.02)$. Neither galantamine nor memantine showed statistically significant differences, but the usage of galantamine was too small for meaningful comparisons. The median retention time of memantine (36.5) compared with that of rivastigmine (14) did appear to indicate longer retention times overall despite the lack of statistical significance ( $\log$ rank $1.5, P<0.44)$. None of the psychiatric medications demonstrated statistical significance when compared with clozapine. The median retention time of quetiapine (46) did appear to reflect tolerability at least equal to that of clozapine when compared with its median retention time (24).

Several other screenings of the data were performed in attempts to define efficacy (Table 3). These included 
Table 2 Retention analysis

\begin{tabular}{lllll}
\hline Drug & Observations $(\mathbf{n})$ & Median \pm IQR & Chi-squared statistics** & P value*** \\
\hline Riv & 72 & $14(3.875-30)$ & Reference & 0.02 \\
Don & 60 & $37.5(5-75.5)$ & 6.2 & NA \\
Gal* & 2 & $25(13-37)$ & NA & 0.44 \\
Mem & 50 & $36.5(4.5-63.25)$ & 1.5 & Reference \\
Clo & 29 & $24(12-77)$ & Reference & 0.76 \\
Que & 109 & $46(18-9 \mid)$ & 0.8 & 0.7 \\
Ola & 7 & $5(3.5-15)$ & 0.9 & \\
\hline
\end{tabular}

Notes: *Inadequate numbers for analysis; **log-rank test; ***adjusted $P$ value using Bonferroni method.

Abbreviations: IQR, interquartile range; Riv, rivastigmine; Don, donepezil; Gal, galantamine; Mem, memantine; Clo, clozapine; NA, not available; Que, quetiapine; Ola, olanzapine.

patient/caregiver and physician perceived benefit when documented. Again, using rivastigmine for cognitive agents and clozapine for antipsychotic agents, we compared the various drugs with their respective gold standard. Olanzapine was the only drug that approached being significantly different (Chi-square $=2.99, P<0.08)$ from the accepted standard. Physician-documented benefit was seen only $43 \%$ of the time and patient/caregiver benefit was only seen $50 \%$ of the time as compared with clozapine, with which benefit was seen $84 \%$ and $95 \%$ of the time, respectively. Because of small numbers, the olanzapine results are of limited generalizability. The other drugs all had documented benefits that were similar in percentage to that seen with the standard. Donepezil actually had a higher percentage of perceived benefit (64\% and 71\%) as compared with rivastigmine (50\% and $65 \%$ ). Quetiapine had a high percentage of perceived documented benefit $(75 \%$ and $78 \%$ ) which was not nearly as robust as for clozapine ( $84 \%$ and 95\%). The Kappa coefficient value demonstrated that the perceived benefit of all the drugs was strongly agreed upon according to the documented responses of both physician and patient/caregiver, with the exception of galantamine, for which the data were insufficient for analysis.

Deaths and side effects were evenly distributed among the different medications, with a few exceptions (Figure 1). Percentages of total side effects ranged from $41.6 \%(5 / 12)$ with olanzapine to $11.4 \%(13 / 114)$ with quetiapine. Death rates ranged from $16.7 \%(2 / 12)$ on olanzapine to $30.0 \%$
(9/30) on clozapine. No agent was associated with a statistically significant higher percentage of death. Weight change was also fairly uniform among the medications, with all medications being within a standard deviation of each other and with no weight change.

\section{Discussion}

Our primary goal was to establish the tolerability of the medications typically used for neuropsychiatric problems in PD by looking at treatment duration in comparison with the accepted gold standard. We defined the gold standard as rivastigmine, the drug approved by the Food and Drug Administration for PD dementia, and clozapine for hallucinations. Both of these agents have previously been shown to be superior to placebo in blinded clinical trials. ${ }^{7,9,15}$ Documented physician benefit and patient/caregiver benefit was further obtained in attempts to demonstrate a perceived therapeutic response of the selected agents.

Our retention results show that no agent was retained significantly less than its respective standard. Donepezil was the only agent that showed statistically significant better retention when compared with rivastigmine, suggesting better tolerability than the standard. However, it should be noted that the rivastigmine group did include patients on both the oral and transdermal form of this medication. The oral form appeared to exhibit a higher degree of gastrointestinal side effects likely leading to a shorter overall duration

Table 3 Proportion of patients with reported benefit

\begin{tabular}{|c|c|c|c|c|c|c|}
\hline \multirow[t]{2}{*}{ Comparison } & \multirow{2}{*}{$\frac{\text { Physician }}{\text { Proportions }}$} & \multirow{2}{*}{$\begin{array}{l}\text { Evaluation } \\
\text { Chi-squared statistics }\end{array}$} & \multirow[t]{2}{*}{$P$ value } & \multirow{2}{*}{$\frac{\text { Patient }}{\text { Proportion }}$} & \multirow{2}{*}{$\begin{array}{l}\text { Evaluation } \\
\text { Chi-squared statistics }\end{array}$} & \multirow[t]{2}{*}{$P$ value } \\
\hline & & & & & & \\
\hline Don versus Riv & $0.64-0.50$ & 1.5 & 0.22 & $0.7 I-0.65$ & 0.11 & 0.74 \\
\hline Gal versus Riv* & NA & NA & NA & $0.67-0.65$ & 0 & 1.00 \\
\hline Mem versus Riv & $0.5 \mathrm{I}-0.50$ & 0 & 1.00 & $0.54-0.65$ & 0.48 & 0.49 \\
\hline Ola versus Clo & $0.43-0.84$ & 2.99 & 0.08 & $0.50-0.95$ & 2.56 & 0.11 \\
\hline Que versus Clo & $0.75-0.84$ & 0.5 & 0.48 & $0.78-0.95$ & 1.66 & 0.20 \\
\hline
\end{tabular}

Note: *No Gal numbers recorded.

Abbreviations: Riv, rivastigmine; Don, donepezil; Gal, galantamine; Mem, memantine; Clo, clozapine; Que, quetiapine; Ola, olanzapine. 


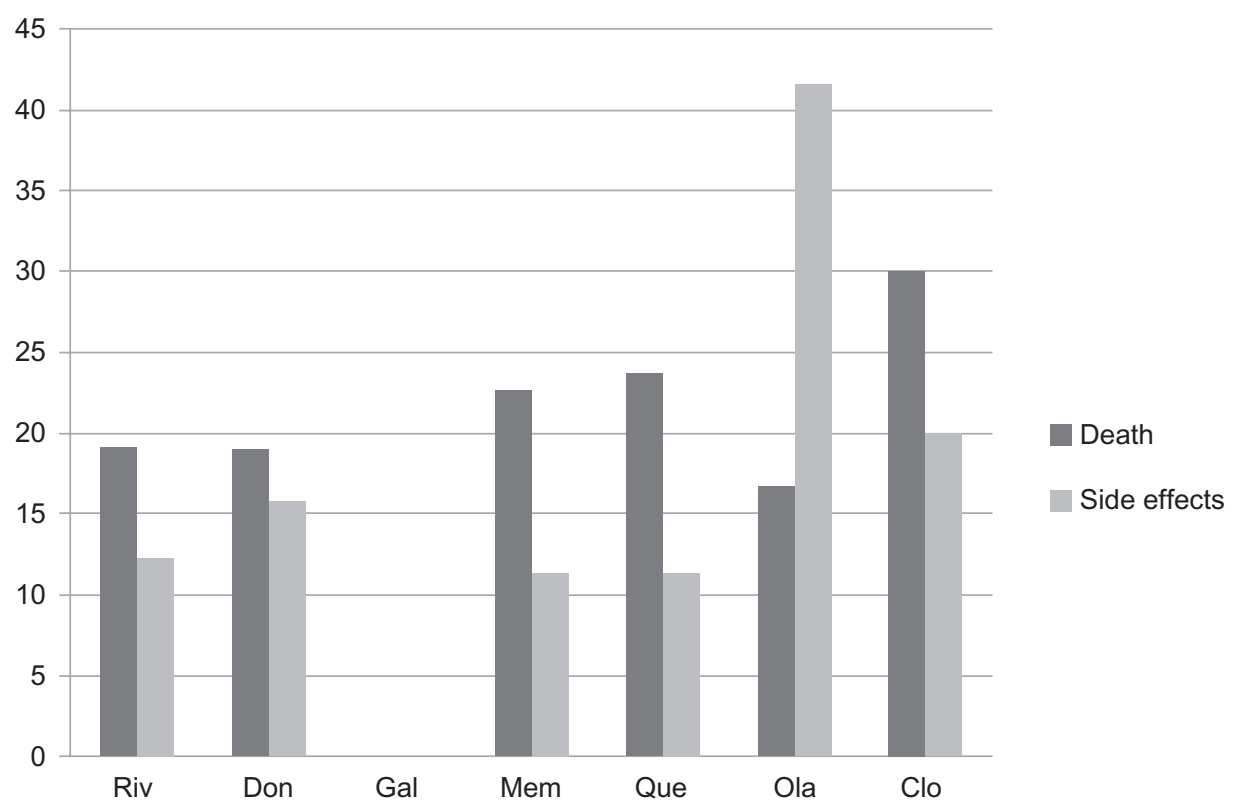

Figure I Percentage of death and side effects.

Abbreviations: Riv, rivastigmine; Don, donepezil; Gal, galantamine; Mem, memantine; Clo, clozapine; Que, quetiapine; Ola, olanzapine.

of therapy. The memantine median was much longer than that for rivastigmine as well, but the interquartile range shows considerable overlap, resulting in a lack of statistical significance. Quetiapine also showed a noticeably larger median retention time than clozapine but also was not statistically significant. Given the concerns about neutropenia and the inconvenience of blood draws for patients, the clozapine results were likely skewed as well, given that the drug was used sparingly late in the disease course in patients who were more severely affected by disease or transiently as an emergent medical treatment.

The documented efficacy data from our database demonstrates that all agents were perceived as at least as effective as the standard medication. Olanzapine was the only agent that approached statistical significance, favoring inferiority. This appears secondary to a high number of side effects, many of which were worsening motor symptoms, a finding that is consistent with prior studies. ${ }^{18,19,25,26}$ Despite not reaching statistical significance, donepezil again seemed to be slightly better than rivastigmine according to these metrics. Quetiapine users also had a large majority (albeit less than clozapine) with an apparent efficacious response, a finding which is also consistent with prior PD studies and would support the aggressive use of this neuroleptic. ${ }^{17}$ It is comforting when looking at the metrics of physician and patient/caregiver documented benefit that the Kappa coefficient is close to one, suggesting a high level of correlation between the two measures.
One could argue that prolonged median retention times reflect not only tolerability but also efficacy, which would support the findings regarding our other metrics. We make some presumptions with this statement, in particular, that ineffective medications would be discontinued by either the clinician or the patient prior to these time points. We believe that clinical pressure to discontinue ineffective medications is supported by many factors: all of these medications are expensive and require some type of monitoring; most patients do not enjoy taking medication, especially those they deem ineffective, because of the burden of pills or patches; and side effects, however mild, generate poor compliance. Use of this metric for efficacy is far from unprecedented. Retention rate has been used successfully in prior neurologic studies as an efficacy measure where the untreated condition can lead to severe morbidity or mortality. ${ }^{21,27-30}$

As for secondary outcome measures, all agents had side effects that occurred at much lower percentages than the percentages of those that achieved benefit. Olanzapine had the highest side effect rate. The duration of treatment until the occurrence of disabling side effects averaged over two years. Death occurred at a consistent rate of about $15 \%-20 \%$ with each medication with the exception of clozapine (30\%). As with our primary outcome measures, there was some concern that this was skewed, given that this agent was used as a last resort in those who were advanced in their illness and had severe neuropsychiatric symptoms. With regards to weight change, there did not appear to be a detectable change, either 
higher or lower. Mean weight change was within one standard deviation of no weight change for every agent.

The conclusion that both atypical neuroleptics and acetylcholinesterase inhibitors as a whole are well tolerated and efficacious in PD contrasts with studies for other neurodegenerative conditions. In Alzheimer's patients, CATIE showed improvement in $26 \%$ of patients on quetiapine and $32 \%$ on olanzapine compared with treatment-limiting side effects in $24 \%$ of those on olanzapine and $16 \%$ of those on quetiapine. ${ }^{21}$ The median time to discontinuation of treatment was 22.1 weeks for olanzapine and 9.1 weeks for quetiapine. ${ }^{21}$ This led the authors to conclude that side effects negated the benefit from atypical neuroleptics. Instead, in our study of PD, we found improvement in $75 \%-78 \%$ of those on quetiapine and $43 \%-50 \%$ on olanzapine, with treatmentlimiting side effects at a rate of $11.4 \%$ (30\% if use at death was included) of those on quetiapine and $41.6 \%$ (58\% if use at death was included) on olanzapine. Further evaluation of deaths was limited due to the fact that the cause of death was frequently unknown. Thus, we could not confirm prior associations of quetiapine with increased cardiovascular morbidity. ${ }^{22}$ It should be noted that most of the patients who died were advanced in age and that the average time of treatment with quetiapine in those on the medication at time of death was 43.38 months. Likewise, the majority of the deaths that occurred on other medications also occurred after an average treatment duration greater than two years as well as in those of advanced age. Without a placebo or other control group, it is difficult to determine the mortality odds from treatment with atypical neuroleptics.

There are several potential concerns with this study. Because of the retrospective nature, recall bias could have affected the response to medications documented in the chart. This was another reason we preferred therapy retention as an additional efficacy measure because it reduced the likelihood of spuriously reported benefit. Critics could certainly argue the merits of retention rate as an efficacy measure but, as stated earlier, this has been established in prior studies as a successful metric. Our method precluded the ability to assess the degree of response as well. There was a definite range reported for those who responded slightly to those who responded more profoundly. We felt creating a placebo group for a potentially more definitive prospective clinical trial evaluating degree and proportion of efficacious response was not practical or ethical given the degree of impairment seen with neuropsychiatric symptoms. Performing objective testing such as the Mini-Mental State Examination or Montreal Cognitive Assessment likely has a place in continuing to explore the efficacy of these medications, but was outside the scope of this study given the lack of relevant data in our database. As a final concern, patients were sometimes lost to follow-up or had large gaps between the end of the observation window and their last visit. This could have had an impact on the data we collected. For example, time on the medication was measured from initiation until the last observation carried forward. Weight was measured from the recorded initial weight until that recorded at the last visit.

In summary, we feel that our study demonstrates effectively that rivastigmine, donepezil, memantine, quetiapine, and clozapine were well tolerated in treating the neuropsychiatric symptoms of PD. Our results support using memantine and the acetylcholinesterase inhibitors for cognitive deficits and the atypical neuroleptics for hallucinations. There is a suggestion that the medicines mentioned above were also efficacious, but only if one accepts the documented physician and patient/ caregiver responses as appropriate metrics, potentially with support from the retention data. Treatment-limiting side effects were substantially less than reported efficacy. Death rate was fairly uniform across the agents, with the exception of clozapine. The overall significance of these deaths is difficult to assess, given our elderly patient population and the prolonged period for which most were on these medications before death. Our results argue that these medications are generally well tolerated and a good option for managing neuropsychiatric problems. Potentially negative outcomes do not appear to offset the perceived clinical benefit from treating these debilitating symptoms.

\section{Disclosure}

The authors report no conflicts of interest in this work.

\section{References}

1. Van Den Eeden S, Tanner C, Bernstein A, et al. Incidence of Parkinson's disease: variation by age, gender, and race/ethnicity. Am J Epidemiol. 2003;157:1015-1022.

2. Miyasaki J. Evidence-based initiation of dopaminergic therapy in Parkinson's disease. Neurology. 2010;257 Suppl 2:S309-S313.

3. Weintraub D, Moberg PJ, Duda JE, Katz IR, Stern MB. Effect of psychiatric and other nonmotor symptoms on disability in Parkinson's disease. J Am Geriatr Soc. 2004;52:784-788.

4. Reichmann H, Schneider C, Lohle M. Non-motor features of Parkinson's disease: depression and dementia. Parkinsonism Relat Disord. 2009; 15 Suppl 3:S87-S92.

5. Rabey J. Hallucinations and psychosis in Parkinson's disease. Parkinsonism Relat Disord. 2009;15 Suppl 3:S105-S110.

6. Aarsland D, Marsh L, Schrag A. Neuropsychiatric symptoms in Parkinson's disease. Mov Disord. 2009;24:2175-2186.

7. Miyasaki JM, Shannon K, Voon V, et al. Practice parameters: evaluation and treatment of depression, psychosis, and dementia in Parkinson disease (an evidence-based review). Report of the Quality Standards Subcommittee of the American Academy of Neurology. Neurology. 2006;66:996-1002. 
8. Ravina B, Putt M, Siderowf A, et al. Donepezil for dementia in Parkinson's disease: a randomized, double-blind, placebo controlled, crossover study. J Neurol Neurosurg Psychiatry. 2005;76:903-904.

9. Emre M, Aarsland D, Albanese A, et al. Rivastigmine for dementia associated with Parkinson's disease. N Engl J Med. 2004;351:2509-2518.

10. Aarsland D, Laake K, Larsen JP, Janvin C. Donepezil for cognitive impairment in Parkinson's disease: a randomised controlled study. J Neurol Neurosurg Psychiatry. 2002;72:708-712.

11. McKeith I, Del Ser T, Spano P, et al. Efficacy of rivastigmine in dementia with Lewy bodies: a randomised, double-blind, placebo-controlled international study. Lancet. 2000;356:2031-2036.

12. van Laar T, De Deyn PP, Aarsland D, Barone P, Galvin JE. Effects of cholinesterase inhibitors in Parkinson's disease dementia: a review of clinical data. CNS Neurosci Ther. 2011;17:428-441.

13. Weiden PJ. EPS profiles: the atypical antipsychotics are not all the same. J Psychiatr Pract. 2007;13:13-24.

14. French Clozapine Study Parkinson Study Group. Clozapine in drug-induced psychosis in Parkinson's disease. Lancet. 1999;353: 2041-2042.

15. Pollack P, Tison F, Rascol O, et al. Clozapine in drug induced psychosis in Parkinson's disease: a randomized, placebo controlled study with open follow up. J Neurol Neurosurg Psychiatry. 2004;75:689-695.

16. The Parkinson Study Group. Low-dose clozapine for the treatment of drug-induced psychosis in Parkinson's disease. $N$ Engl J Med. 1999;340:757-763.

17. Shotbolt P, Samuel M, David A. Quetiapine in the treatment of psychosis in Parkinson's Disease. Ther Adv Neurol Disord. 2010;3:339-350.

18. Breier A, Sutton VK, Feldman PD, et al. Olanzapine in the treatment of dopamimetic-induced psychosis in patients with Parkinson's disease. Biol Psychiatry. 2002;52:438-445.

19. Ondo W, Levy JK, Vuong KD, Hunter C, Jankovic J. Olanzapine treatment for dopaminergic-induced hallucinations. Mov Disord. 2002;17:1031-1035.

20. Goetz CG, Blasucci LM, Leurgans S, Pappert EJ. Olanzapine and clozapine: comparative effects on motor function in hallucinating PD patients. Neurology. 2000;55(6):789-794.
21. Schneider L, Tariot PN, Dagerman KS, et al. Effectiveness of atypical antipsychotic drugs in patients with Alzheimer's Disease. $N$ Engl $J$ Med. 2006;355:1525-1538.

22. Daumit G, Goff D, Meyer JM, et al. Antipsychotic effects on estimated 10-year coronary heart disease risk in the CATIE schizophrenia study. Schizophr Res. 2008;105:175-187.

23. Zheng L, Mack WJ, Dagerman KS, et al. Metabolic changes associated with second-generation antipsychotic use in Alzheimer's disease patients: the CATIE-AD study. Am J Psychiatry. 2009;166:1063-1064.

24. Schneider L, Dagerman KS, Insel P. Risk of death with atypical antipsychotic drug treatment for dementia: meta-analysis of randomized placebo-controlled trials. JAMA. 2005;294:1934-1943.

25. Beasley CM Jr, Tollefson G, Tran P, Satterlee W, Sanger T, Hamilton S. Olanzapine versus placebo and haloperidol: acute phase results of the North American double-blinded olanzapine trial. Neuropsychopharmacology. 1996;14:111-123.

26. Ghaemi SN, Hsu DJ, Rosenquist KJ, Pardo TB, Goodwin FK. Extrapyramidal side effects with atypical antipsychotics in bipolar disorder. Prog Neuropsychopharmacol Biol Psychiatry. 2006;30: 209-213.

27. $\mathrm{Hu} \mathrm{Y,} \mathrm{Lu} \mathrm{Y,} \mathrm{Yu} \mathrm{W,} \mathrm{et} \mathrm{al.} \mathrm{Long-term} \mathrm{retention} \mathrm{rate} \mathrm{of} \mathrm{topiramate} \mathrm{as}$ initial monotherapy in Chinese patients with newly diagnosed epilepsy: a prospective, observational study. Epilepsy Res. 2010;90:278-284.

28. Yuen AW, Singh R, Bell GS, et al. The long-term retention of pregabalin in a large cohort of patients with epilepsy at a tertiary referral centre. Epilepsy Res. 2009;87:120-123.

29. Arif H, Buchsbaum R, Pierro J, et al. Comparative effectiveness of 10 antiepileptic drugs in older adults with epilepsy. Arch Neurol. 2010;67:408-415.

30. Carpay JA, Aalbers K, Graveland GA, Engelsman M. Retention of new AEDs in institutionalized intellectually disabled patients with epilepsy. Seizure. 2009; 18:119-123.
Journal of Parkinsonism \& Restless Legs Syndrome

\section{Publish your work in this journal}

Journal of Parkinsonism and Restless Legs Syndrome is an online, open access, peer-reviewed journal. The journal publishes review articles, historical reviews, original research articles, case reports, letters to the editor, clinical teaching cases, neuroradiology highlights, neuropathology highlights, neuropsychiatry highlights, autobiographies, conference

\section{Dovepress}

proceedings, abstracts and book reviews. The manuscript management system is completely online and includes a very quick and fair peerreview system, which is all easy to use. Visit http://www.dovepress.com/ testimonials.php to read real quotes from published authors. 